\title{
INFECTION WITH MICROCOCCUS TETRAGENUS AS A COMPLICATION OF PENETRATING KERATOPLASTY*
}

\author{
BY \\ G. J. ROMANES \\ From the Corneo-Plastic Unit and Regional Eye Bank, Queen Victoria Hospital, East \\ Grinstead, Sussex
}

EXPERIENCE in a recent case shows many points of interest.

\section{Case Report}

A male medical student, aged 22, had bilateral keratoconus which had been successfully treated by Mr. H. Treissman with contact lenses for 7 years. Recently the left eye had become intolerant to the contact glass with recurrent corneal ulceration and scar formation. The patient was referred to Mr. B. W. Rycroft for consideration of keratoplasty.

History.-There were no points of relevant ophthalmic interest in the general health or family history. There was no evidence of closed focal sepsis and a routine radiological examination of the chest was normal.

Examination.-Visual acuity: right eye with contact lens 6/12 (ii); left eye with contact lens $1 / 60$.

Projection of light was accurate and brisk in each eye to a dull stimulus.

The intra-ocular pressure was normal. Marked cone formation was present on each cornea with typical ring distortion and concentric concentration was seen on the keratoscope. There was fluorescein stain on the left cornea limited to the apex of the cone about $3 \mathrm{~mm}$. in diameter; it was surrounded by a circle of punctate staining (Fig. 1).

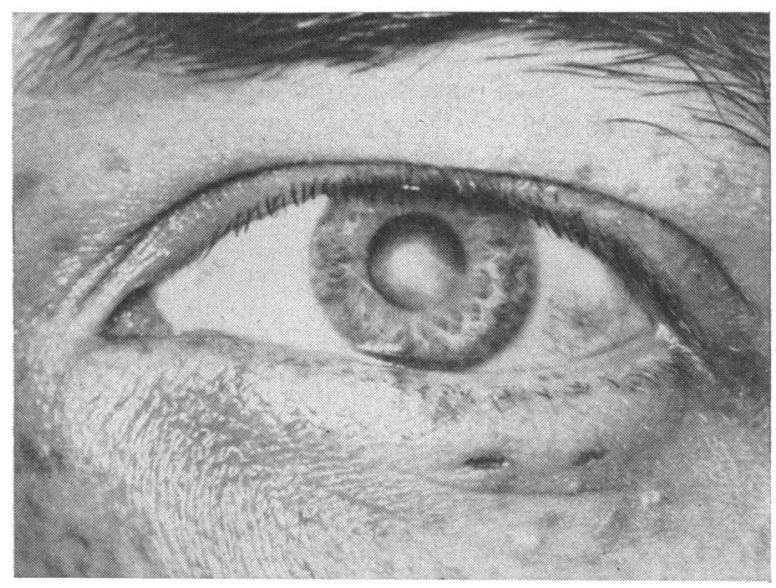

Fig. 1.-Left eye on admission.

Slit-Lamp Report (Left eye).-The epithelium was regular at the periphery, but in the central region there was marked irregularity with some opacity. The contour of the anterior surface was obviously conical with the apex slightly down and in from the corneal centre.

* Received for publication August 9, 1957. 
The stroma had normal thickness above and below at the periphery, but there was a horizontal thin area across the cornea. In the cone the total stromal thickness was reduced by 70 per cent. compared to the periphery above and below.

The posterior corneal surface showed marked irregularity with linear wrinkling of Descemet's membrane in the base of the cone. There were no cells adherent to the posterior corneal surface.

The anterior chamber was increased in depth in the centre, and was normal at the periphery; no flare was present. The iris pattern and movements were normal. The lens capsule and anterior lens cortex were normal. The fundus showed no abnormality with the ophthalmoscope.

Plan.-Because of recurrent ulceration, diminished contact lens tolerance, deterioration of visual acuity, and the progressive nature of the disease, a left penetrating keratoplasty was advised.

Donor Material.-This was a fresh eye, excised with full aseptic precautions 2 hours previously for a choroidal neoplasm, and suspended in saline vapour in the refrigerator at $+4^{\circ} \mathrm{C}$. This eye had been treated for 48 hours before operation with chloramphenicol 1 per cent. ointment. An overnight pad on the donor eye showed no.stickiness and a control 48-hour culture of the conjunctiva was sterile.

Operation.-On August 28, 1956, an 8-mm. penetrating keratoplasty (B. W. R. and G. J. R.) was performed under local anaesthesia; intravenous potentialization was obtained with Pethidine, Phenergan, and Largactil (dosage $50 \mathrm{mg}$. of each).

The graft was secured by four direct sutures in the vertical and horizontal meridians, reinforced with two oblique overlay sutures across sterile egg membrane. At the end of the operation a subconjunctival injection of $10 \mathrm{mg}$. cortisone was given. Ung. chloramphenicol 1 per cent. and ung. eserine 0.5 per cent. were applied after the anterior chamber had reformed. Air was not insufflated. Double pad dressings were applied with a pressure bandage over the left eye.

Post-operative Course.-There was a little vomiting in the first part of the week but there was no undue discomfort in the eyes. The first dressing was done on the seventh day, as is the routine custom. The pad was sticky with a yellowish-green purulent material and there was excessive swelling of the lids. The lower lid Frost suture was under tension due to the oedema of the lids. After local anaesthesia, the eye was seen to be red with gross conjunctival infection and chemosis; one cruciate suture had cut out in the infra-nasal region. The cruciate sutures and egg membrane were removed and pus was adherent to the direct sutures; the graft showed infection of the infra-nasal quadrant extending almost to its centre, and the 6 and 9 o'clock direct sutures had also cut out. There was also gross infection of the host stroma with a 2-mm. hypopyon. The pupil was in miosis with many posterior synechiae.

A swab was taken, an immediate subconjunctival injection of streptomycin and penicillin was made, and "Polyfax" ointment was instilled. Local intensive antibiotic therapy was immediately commenced with a drop of streptomycin $50 \mathrm{mg} . / \mathrm{ml}$. every minute.

Culture Report.-A pure growth of Micrococcus tetragenus sensitive only to chloramphenicol was found.

\footnotetext{
"Micrococcus tetragenus may occur as a commensal on the mucous membrane of the upper respiratory passages; it has also been found on the skin. It is often found in suppurations in the region of the mouth or in the neck, e.g. dental abscess, and also occurs in various lesions of the respiratory tract, in phthisical cavities, abscesses in the lungs, etc. Sometimes it is present alone, and probably has a pyogenic action in the human subject under certain conditions. In most cases it is associated with other organisms. Cases of general infection along with pneumonic symptoms have been recorded, the organism having been isolated from the blood; recovery was the rule. Cases of pyaemia have also been described in which this
} 
organism was found in a state of purity in the pus in various situations." (Browning and Mackie, 1949.)

An examination under general anaesthesia was made the next day, and it was found that the union of the host-graft junction was weak and primary ectasia of the graft had set in. During a subconjunctival injection of saturated solution of penicillin and streptomycin, the wound gave way below with loss of the anterior chamber. To reinforce this rupture a conjunctival "bucket-handle" flap was immediately fashioned and secured over the bulging section to provide cover and additional blood supply. When the flap was removed a week later the union was seen to be firm again.

On the 8th day there was a small leucocytosis. Daily subconjunctival chloramphenicol and mydricaine injections were continued for 8 days under light general anaesthesia. During the whole of this period chloramphenicol $250 \mathrm{mg}$. daily was given by mouth, and injections of streptomycin $1 \mathrm{~g}$. daily and penicillin $1 \mathrm{M}$ units were given daily. Chloramphenicol drops were instilled at 5-minute intervals day and night. Phenylephrine 10 per cent. was used for the first 2 days; thereafter it was discontinued and gutt. cortisone $5 \mathrm{mg}$. $/ \mathrm{ml}$. were started. Atropine 1 per cent. drops were used every $4 \mathrm{hrs}$ throughout.

On the tenth day the total white cell count had fallen to normal, indicating that the infection had now responded to the antibiotics.

The clinical appearance showed progressive changes of improvement from a state of gross infection with hypopyon to a final stage of a white eye with a small colarette anterior synechiae in the inferior nasal quadrant. There remained little more than peripheral fibrosis in the graft; the central area was clear and there was minimal residual opacity in the adjacent host tissue.

The visual acuity in the left eye was counting fingers at $1 \mathrm{~m} .4$ weeks after operation and in the seventh week it was 6/36 unaided, and the patient was discharged from hospital.

Post-operative Visual Acuity. -6 weeks later the visual acuity in the left eye without correction was 6/18, and the eye was white and quiet. A contact lens trial 32 weeks after operation resulted in a visual acuity of $6 / 9$ and the tolerance was excellent (Fig. 2).

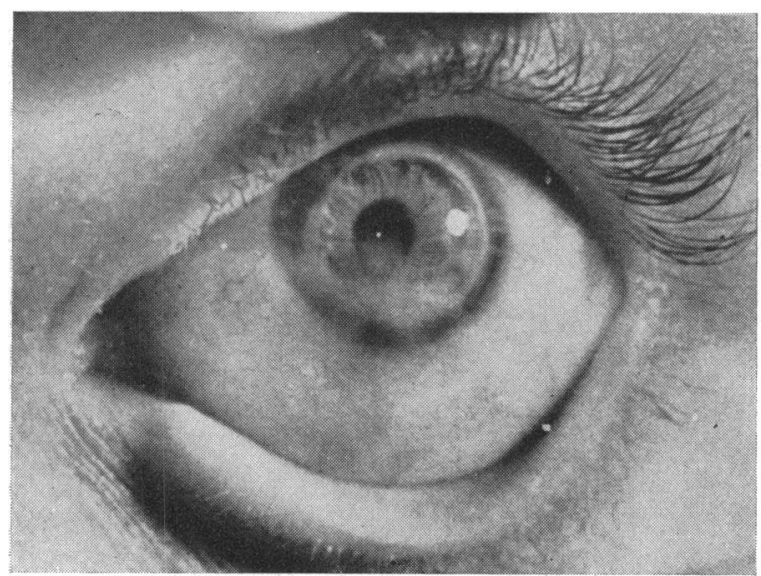

Fig. 2.-Left eye 32 weeks after operation.

\section{Comment}

This case represents one of only two infected grafts in over 300 graft cases at this Unit. The following points are of interest:

(1) A fresh donor eye, in spite of previous intensive treatment by antibiotics, 
may be a potential source of infection owing to chance resistance. It is not possible, however, to define the exact source of infection in this case, but the initial site appeared to be in the graft. The organism rarely causes pyaemic lesions and is usually considered to be a commensal in an established respiratory lesion. The theatre is air-conditioned and has regular bacteriological control.

(2) Early bacteriological diagnosis and establishment of sensitivity enabled appropriate antibiotic therapy to be given at the most opportune time.

(3) The opinion of our pathologist, Dr. A. Sachs, that little fibrotic reaction would result from this particular organism has been fully justified. The visual acuity improved from $1 / 60$ to $6 / 9$.

(4) Intensive and continuous local treatment, coupled with devoted and enlightened nursing, were the factors of prime importance in the successful outcome. "Minute drops" were used for $24 \mathrm{hrs}$, and one drop every 5 minutes for 3 days and nights.

(5) The prognosis of severe intra-ocular post-operative infection need not necessarily be catastrophic to vision, provided skilled team-work is available.

I wish to acknowledge the help and encouragement I have had in the preparation of this report from Mr. B. W. Rycroft and Dr. A. Sachs, and to place on record the devoted care of Sisters King and Nuthall and the nursing staff of the Corneo-Plastic Unit. I am indebted to Gordon Clementson for the photographs.

\section{REFERENCE}

BrownIng, C. H., and MackIE, T. J. (1949). “Text-book of Bacteriology”, 11th ed. of Muir and Ritchie's "Manual of Bacteriology", pp. 149, 150. Oxford University Press, London. 